\title{
Collective and single-particle excitations of a trapped Bose gas
}

\author{
F. Dalfovo, ${ }^{1}$ S. Giorgini, ${ }^{2}$ M. Guilleumas, ${ }^{1}$ L. Pitaevskii, ${ }^{3,4}$ and S. Stringari ${ }^{1}$ \\ ${ }^{1}$ Dipartimento di Fisica, Università di Trento and Istituto Nazionale Fisica della Materia, I-38050 Povo, Italy \\ ${ }^{2}$ European Centre for Theoretical Studies in Nuclear Physics and Related Areas, Villa Tambosi, I-38050 Villazzano, Italy \\ ${ }^{3}$ Department of Physics, TECHNION, Haifa 32000, Israel \\ ${ }^{4}$ Kapitza Institute for Physical Problems, ulica Kosygina 2, 117334 Moscow, Russia
}

(Received 21 May 1997)

\begin{abstract}
The density of states of a Bose-condensed gas confined in a harmonic trap is investigated. The predictions of Bogoliubov theory are compared with those of Hartree-Fock theory and of the hydrodynamic model. We show that the Hartree-Fock scheme provides an excellent description of the excitation spectrum in a wide range of energy, revealing a major role played by single-particle excitations in these confined systems. The crossover from the hydrodynamic regime, holding at low energies, to the independent-particle regime is explicitly explored by studying the frequency of the surface mode as a function of their angular momentum. The applicability of the semiclassical approximation for the excited states is also discussed. We show that the semiclassical approach provides simple and accurate formulas for the density of states and the quantum depletion of the condensate. [S1050-2947(97)05111-1]
\end{abstract}

PACS number(s): 03.75.Fi, 67.40.Db

\section{INTRODUCTION}

The collective modes of a Bose-condensed gas confined by an external potential have been the object of extensive work in the last months. The successful agreement between experimental results $[1,2]$ and theoretical predictions [3-8] for the collective frequencies at low temperature has stimulated intensive research activity. Though only the modes with low multipolarity and frequency have been detected in experiments, the excitations at higher energy and angular momentum are also very important because they determine the statistical behavior of the system, including thermodynamics, transport phenomena, and superfluid effects.

The excited states at high energy are expected to have single-particle nature. However, the transition from collective (phononlike) to single-particle excitations in an inhomogeneous system can differ significantly from the case of a uniform Bose gas. In fact, the presence of a surface allows for the occurrence of single-particle states even at low energy (lower than the chemical potential). These states, of low energy but high multipolarity, are localized near the surface, where the condensate density becomes small. This behavior represents a peculiar and interesting feature of these confined systems; in a uniform Bose gas, in fact, only phonons are present at low energy. In a recent paper [9] we have already pointed out the effects of these single-particle states on the thermodynamic properties of the trapped gases.

In the present work we solve the equations for the excited states of a weakly interacting gas in a spherical trap at zero temperature within Bogoliubov theory. The main purpose is to investigate the collective (phononlike) and single-particle character of the elementary excitations. This is accomplished by calculating key quantities, such as the density of states, the frequency of the surface modes, and the quantum depletion of the condensate, and by comparing the predictions of Bogoliubov theory with the ones of different approximations, like Hartree-Fock theory and the hydrodynamic model. Finally we check the accuracy of the semiclassical approxi- mation and we show that it provides simple and useful formulas for both the density of states and the quantum depletion of the condensate.

\section{BOGOLIUBOV THEORY}

The elementary excitations of a degenerate Bose gas are associated with the fluctuations of the condensate. At low temperature they are described by the time-dependent GrossPitaevskii (GP) equation for the order parameter [10]:

$$
i \hbar \frac{\partial}{\partial t} \Psi(\mathbf{r}, t)=\left(-\frac{\hbar^{2} \nabla^{2}}{2 m}+V_{\text {ext }}(\mathbf{r})+g|\Psi(\mathbf{r}, t)|^{2}\right) \Psi(\mathbf{r}, t),
$$

where $\int d \mathbf{r}|\Psi|^{2}=N$ is the number of atoms in the condensate. At zero temperature $N$ coincides with the total number of atoms, except for a very small difference $\delta N \ll N$ due to the quantum depletion of the condensate. The coupling constant $g$ is proportional to the $s$-wave scattering length $a$ through $g=4 \pi \hbar^{2} a / m$. In the present work we will discuss the case of positive scattering length, $a>0$, as in the experiments with rubidium and sodium, but the same formalism can be also applied to systems with negative scattering length. The trap is included through $V_{\text {ext }}$, which is chosen here in the form of an isotropic harmonic potential: $V_{\text {ext }}(r)=(1 / 2) m \omega_{\mathrm{HO}}^{2} r^{2}$. The harmonic trap provides also a typical length scale for the system, $a_{\mathrm{HO}}=\left(\hbar / m \omega_{\mathrm{HO}}\right)^{1 / 2}$. Actually, the experimental traps have cylindrical symmetry, with different radial and axial frequencies, but the choice of a spherical trap, as we will discuss later, is not expected to affect the main conclusions of the present work, while reducing greatly the numerical effort.

The normal modes of the condensate can be found by linearizing the GP equations, i.e., looking for solutions of the form 


$$
\Psi(\mathbf{r}, t)=e^{-i \mu t / \hbar}\left[\Psi_{0}(\mathbf{r})+u(\mathbf{r}) e^{-i \omega t}+v^{*}(\mathbf{r}) e^{i \omega t}\right],
$$

where $\mu$ is the chemical potential and functions $u$ and $v$ are the "particle" and "hole" components characterizing the Bogoliubov transformations. After inserting in Eq. (1) and retaining terms up to first order in $u$ and $v$, one finds three equations. The first one is the nonlinear equation for the order parameter of the ground state [10],

$$
\left[H_{0}+g \Psi_{0}^{2}(\mathbf{r})\right] \Psi_{0}(\mathbf{r})=\mu \Psi_{0}(\mathbf{r})
$$

where $H_{0}=-\left(\hbar^{2} / 2 m\right) \nabla^{2}+V_{\text {ext }}(\mathbf{r})$, while $u(\mathbf{r})$ and $v(\mathbf{r})$ obey the following coupled equation:

$$
\begin{aligned}
& \hbar \omega u(\mathbf{r})=\left[H_{0}-\mu+2 g \Psi_{0}^{2}\right] u(\mathbf{r})+g \Psi_{0}^{2} v(\mathbf{r}), \\
& -\hbar \omega v(\mathbf{r})=\left[H_{0}-\mu+2 g \Psi_{0}^{2}\right] v(\mathbf{r})+g \Psi_{0}^{2} u(\mathbf{r}) .
\end{aligned}
$$

Numerical solutions of these equations have been recently found by different authors [4-8]. In the present work, we use them to calculate the density of states, the frequency of the surface modes, and the quantum depletion of the condensate, in order to clarify the different roles played by excitations having collective and single-particle character.

When the dimensionless parameter $N a / a_{\mathrm{HO}}$ is large, the kinetic energy term in the ground-state equation (3) becomes negligible with respect to the mean-field term and one gets the Thomas-Fermi approximation:

$$
\Psi_{0}^{\mathrm{TF}}(\mathbf{r})=\left(\frac{\mu^{\mathrm{TF}}-V_{\text {ext }}(\mathbf{r})}{g}\right)^{1 / 2},
$$

with

$$
\mu^{\mathrm{TF}}=\frac{\hbar \omega_{\mathrm{HO}}}{2}\left(15 \frac{N a}{a_{\mathrm{HO}}}\right)^{2 / 5}
$$

In the same limit the equations of motion (4) and (5) coincide with the equations of the hydrodynamics (HD) of superfluids $[3,11,12]$. In the spherical case their eigenfrequencies take the analytic form [3]

$$
\omega(n, \ell)=\omega_{\mathrm{HO}}\left(2 n^{2}+2 n \ell+3 n+\ell\right)^{1 / 2},
$$

where $\ell$ and $n$ are the angular momentum quantum number and the number of nodes in the radial solution, respectively. The deviations from the predictions of the noninteracting harmonic oscillator (HO) model,

$$
\omega(n, \ell)=\omega_{\mathrm{HO}}(2 n+\ell),
$$

point out the effects of two-body interactions. These are particularly important for the so-called "surface" modes $(n=0)$, where the HO prediction $\omega=\ell \omega_{\mathrm{HO}}$ is significantly lowered to the hydrodynamic value $\omega=\sqrt{\ell} \omega_{\mathrm{HO}}$. In general the HD prediction turns out to be very accurate for the lowenergy excitations of the system, while the ideal gas prediction is expected to be valid in the opposite case of high excitation energies. The exact solutions of Eqs. (4) and (5) provide the correct interpolation between the two limiting regimes.

A typical spectrum obtained from Eqs. (4) and (5) is given in the upper part of Fig. 1 for a gas of $N=10000$

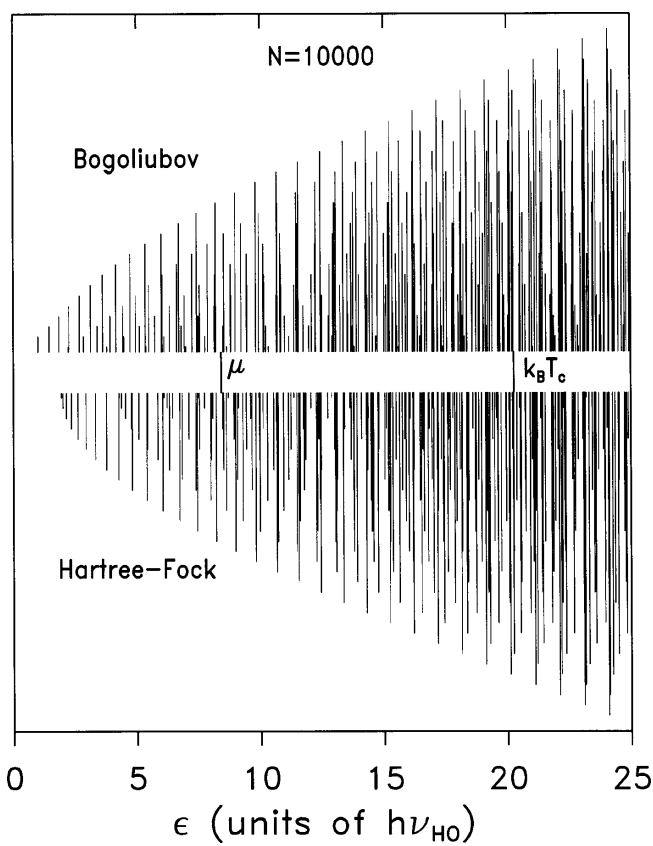

FIG. 1. Excitation spectrum of 10000 atoms of ${ }^{87} \mathrm{Rb}$ in a spherical trap with $a_{\mathrm{HO}}=0.791 \times 10^{-4} \mathrm{~cm}$. The vertical bars have length $(2 \ell+1)$. The upper spectrum corresponds to the numerical solution of Eqs. (4) and (5); the lower one is the spectrum of the Hartree-Fock Hamiltonian (11). Two energy scales are also shown in the figure: the chemical potential $\mu=8.41$, fixed by the solution of Eq. (3), and the critical temperature for a noninteracting gas in the same trap, $k_{B} T_{c}=20.26$.

atoms of rubidium (scattering length $a=110 a_{0}$, where $a_{0}$ is the Bohr radius). For the spherical trap we have chosen the frequency $\omega_{\mathrm{HO}}=2 \pi \nu_{\mathrm{HO}}=2 \pi 187 \mathrm{~Hz}$, which is the average $\omega_{\mathrm{HO}}=\left(\omega_{x} \omega_{y} \omega_{z}\right)^{1 / 3}$ of the axial and radial frequencies of Ref. [1]. It corresponds to the oscillator length $a_{\mathrm{HO}}=0.791 \times 10^{-4} \mathrm{~cm}$. Energy is given in units $\hbar \omega_{\mathrm{HO}}$ and the chemical potential is 8.41 in these units. The vertical bars have length $(2 \ell+1)$, so that the angular momentum of each state can be inferred from the figure. One clearly sees that, at energy much larger than the chemical potential, the excited states tend to be grouped into levels $\hbar \omega_{\mathrm{HO}}$ apart, as in the noninteracting HO model. Conversely, the energy of the lowest modes is close to the prediction of the HD equations [3]. For instance, the lowest $\ell=2$ and $\ell=0$ modes differ by less than $3 \%$ from the hydrodynamic values $\sqrt{2}$ and $\sqrt{5}$, respectively.

\section{DENSITY OF STATES}

Once the spectrum of excited states is calculated, one can count the number of states below a given energy $\epsilon$ :

$$
\mathfrak{N}(\epsilon)=\sum_{\hbar \omega<\epsilon}(2 \ell+1)
$$

The density of states $g(\epsilon)$ is simply the derivative $d \mathfrak{N}(\epsilon) / d \epsilon$. Note that the quantity $\mathfrak{N}(\epsilon)$ is well defined even for a discretized spectrum, while the density of states implies averaging the number of states within small but finite energy intervals.

In Fig. 2 we show the quantity $\mathfrak{N}(\epsilon)$ obtained by counting the levels in the spectrum of Fig. 1. For comparison the 


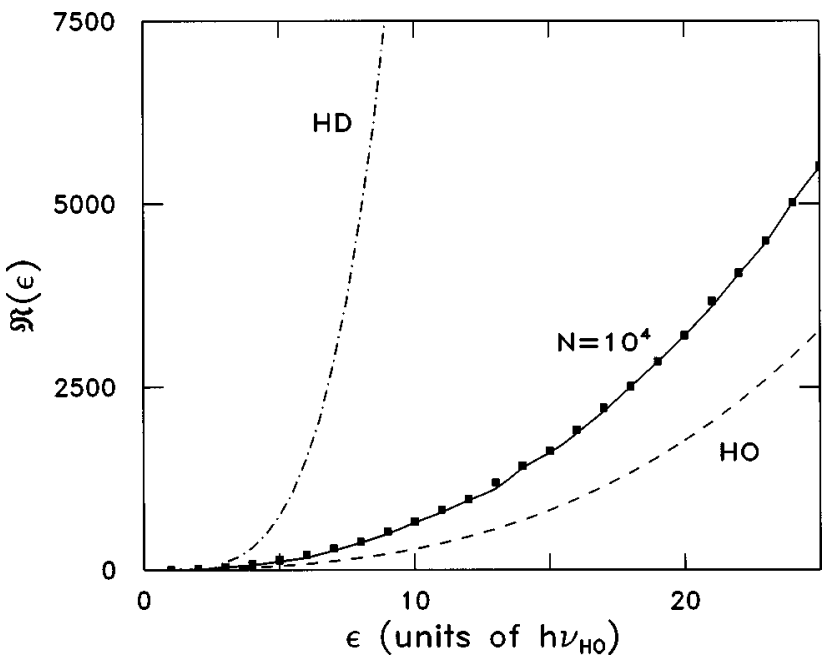

FIG. 2. Number of states $\mathfrak{N}(\epsilon)$ vs energy. The Bogoliubov (points) and Hartree-Fock (solid line) predictions, obtained by counting the states in Fig. 1, are compared with the ones of the noninteracting harmonic oscillator (dashed line) and of hydrodynamic equations (dot-dashed line).

results of the noninteracting harmonic oscillator (9) and of the hydrodynamic model (8) are also shown. The effects of the repulsive interatomic forces are clearly responsible for an enhancement of the density of states with respect to the ideal gas. However $\mathfrak{N}(\epsilon)$ remains well below the HD approximation, the latter being soon inadequate as $\epsilon$ increases. Indeed hydrodynamic theory accounts for collective phenomena and provides an excellent description of the low-lying elementary excitations of the system [3,8], but completely ignores single-particle effects. This is exactly the opposite of what Hartree-Fock (HF) theory does. For this reason it is interesting to compare the results of Bogoliubov theory with the predictions of HF theory in which one determines the eigenstates of the single-particle Hamiltonian $[13,14]$

$$
H_{\mathrm{HF}}=-\left(\hbar^{2} / 2 m\right) \nabla^{2}+V_{\mathrm{ext}}(\mathbf{r})-\mu+2 g \Psi_{0}^{2}(\mathbf{r}) .
$$

The lowest eigenstates of the HF Hamiltonian are expected to be localized near the surface of the condensate. To understand this point better, let us take the large- $N$ limit. In this case one can use the Thomas-Fermi approximation (6) for the ground-state density. The HF Hamiltonian then takes the simple form

$$
H_{\mathrm{HF}}=-\left(\hbar^{2} / 2 m\right) \nabla^{2}+\frac{1}{2} m \omega_{\mathrm{HO}}^{2}\left|r^{2}-R^{2}\right|,
$$

where $R=\left[2 \mu^{\mathrm{TF}} /\left(m \omega_{\mathrm{HO}}^{2}\right)\right]^{1 / 2}$ is the classical radius of the condensate. The HF potential has a pronounced minimum at $R$. This potential well near the boundary persists in the HF Hamiltonian even for smaller values of $N$.

It is worth stressing that, in general, Hartree-Fock theory is expected to be correct for energies larger than the chemical potential. For these trapped bosons, however, it accounts also for the low-energy excitations close to the boundary, where the density of the condensate is small. This can be seen in Fig. 1, where the Hartree-Fock and Bogoliubov spectra are compared. One notes that the two spectra are rather similar even below the chemical potential $(\mu=8.41$ in this case)

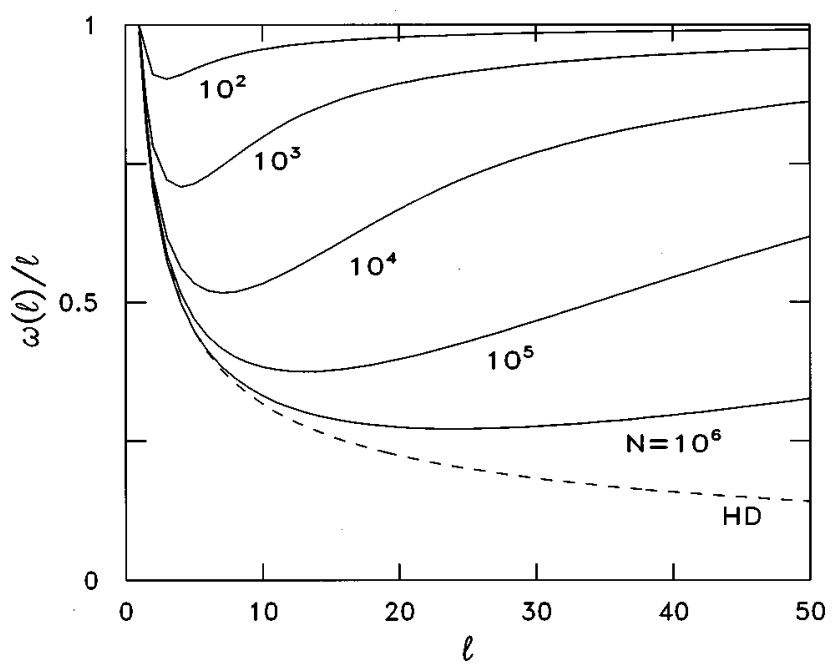

FIG. 3. Frequency (in units of $\omega_{\mathrm{HO}}$ ) of the $n=0$ excited states as a function of their angular momentum $\ell$ for $N$ atoms of ${ }^{87} \mathrm{Rb}$ in a spherical trap with $a_{\mathrm{HO}}=0.791 \times 10^{-4} \mathrm{~cm}$. The hydrodynamic prediction is shown as a dashed line.

except for the excitations having lowest angular momenta, i.e., shortest bars in the figure. Those collective excitations are instead correctly reproduced by the hydrodynamic model. The corresponding Hartree-Fock prediction for the quantity $\mathfrak{N}(\epsilon)$ is also given in Fig. 2 as a solid line. The agreement with the results of Eqs. (4) and (5) is remarkable in the whole range of energy. The figure shows the case of 10000 atoms but a similar agreement is found for all values of $N$ relevant for the experiments $\left(N \simeq 10^{3}-10^{7}\right)$. The above behavior represents a major difference with respect to the case of a homogeneous Bose gas where phonons have a crucial effect on the density of states.

\section{SURFACE EXCITATIONS}

In order to better understand the transition from the collective to the single-particle regime, we have explored in detail the evolution of the excitation energy for the surface modes $(n=0)$ as a function of their angular momentum $\ell$, as predicted by the solution of Eqs. (4) and (5). As already mentioned, the effects of the interaction are particularly important for such modes and are responsible for a significant lowering of their frequency. In Fig. 3 we plot the quantity $\omega / \ell$, in units $\omega_{\mathrm{HO}}$, for different numbers of atoms in the same trap. This ratio has an important physical meaning because, according to Landau's criterium for superfluidity, it provides the rotational frequency at which the $\ell$ th surface excitation becomes unstable. The hydrodynamic prediction (8) is shown as a dashed line, the curve going asymptotically to zero for large $\ell$. The figure shows that the Bogoliubov states first follow the HD curve, but, rather soon, they deviate from it, approaching asymptotically the noninteracting value $\omega_{\ell} / \ell=\omega_{\mathrm{HO}}$. The deviation from HD takes place at larger values of $\ell$ if $N$ is increased, revealing that the HD approximation becomes applicable to a larger number of states in this limit.

A simple estimate of the typical value of $\ell$ at which the HD picture starts failing, one can take [15] $\ell_{c} \simeq R p_{c}$ where $R$ is the radius of the condensate, proportional to $N^{1 / 5}$, and 


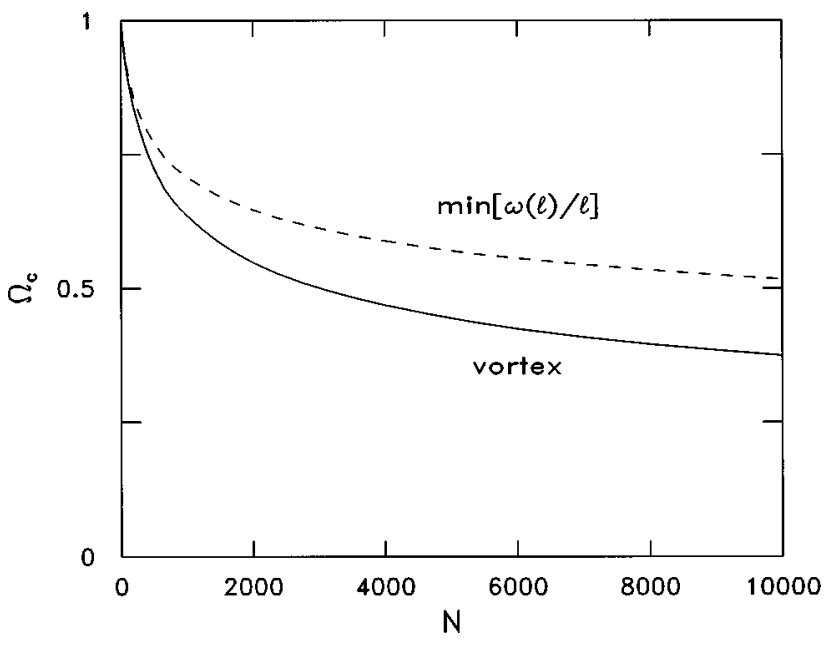

FIG. 4. Critical rotational frequency (in units of $\omega_{\mathrm{HO}}$ ) for producing a quantized vortex (solid line) or surface states (dashed line) as a function of the number of rubidium atoms in the spherical trap.

$p_{c}$ is of the order of the inverse of the surface thickness $d=\left[a_{\mathrm{HO}}^{4} /(2 R)\right]^{1 / 3}[16-18]$. For larger values of $\ell$, the wavelength of the excitations becomes shorter than $d$ and one explores microscopic details of the boundary that cannot be described by the Thomas-Fermi approximation (6) and by the HD equations. This yields $l_{c} \propto\left(R / a_{\mathrm{HO}}\right)^{4 / 3} \propto N^{4 / 15}$, corresponding to an excitation energy $\hbar \omega \propto N^{2 / 15}$, smaller than the chemical potential, which instead behaves as $N^{2 / 5}$. This explains why the crossover from the HD to the single-particle regime takes place at energies smaller than $\mu$.

For each value of $N$, the curves in Fig. 3 exhibit minima and one can define a critical frequency as $\Omega_{c}=\min (\omega / \ell)$. For rotational frequencies larger than $\Omega_{c}$ the surface excitations become unstable. It is interesting to compare this value with the critical frequency needed to generate a vortex [19]. This is done in Fig. 4 where we compare the two critical frequencies as a function of $N$. We find that the lowest instability is always associated with the creation of a vortex. Note, however, that in order to generate a vortex one needs to transfer to the system a huge angular momentum (equal to $N \hbar$ ), which is much higher than the value $\ell$ required to create a surface excitation.

\section{SEMICLASSICAL APPROXIMATION AND SCALING BEHAVIOR}

A good approximation for the density of states can be obtained by solving Eqs. (4) and (5) in the semiclassical approximation $[9,20,21]$. In this approximation, which is expected to hold for excitation energies much larger than the oscillator energy $\hbar \omega_{\mathrm{HO}}$, the quantity $\mathfrak{N}(\epsilon)$ is a continuous function of $\epsilon$ defined by

$$
\mathfrak{N}(\epsilon)=\int_{0}^{\epsilon} d \epsilon^{\prime} \int \frac{d \mathbf{r} d \mathbf{p}}{(2 \pi \hbar)^{3}} \delta\left(\epsilon^{\prime}-\epsilon(\mathbf{r}, \mathbf{p})\right)
$$

where

$$
\boldsymbol{\epsilon}(\mathbf{r}, \mathbf{p})=\left[\left(\frac{p^{2}}{2 m}+V_{\mathrm{ext}}(\mathbf{r})-\mu+2 g n_{0}(\mathbf{r})\right)^{2}-g^{2} n_{0}^{2}(\mathbf{r})\right]^{1 / 2}
$$
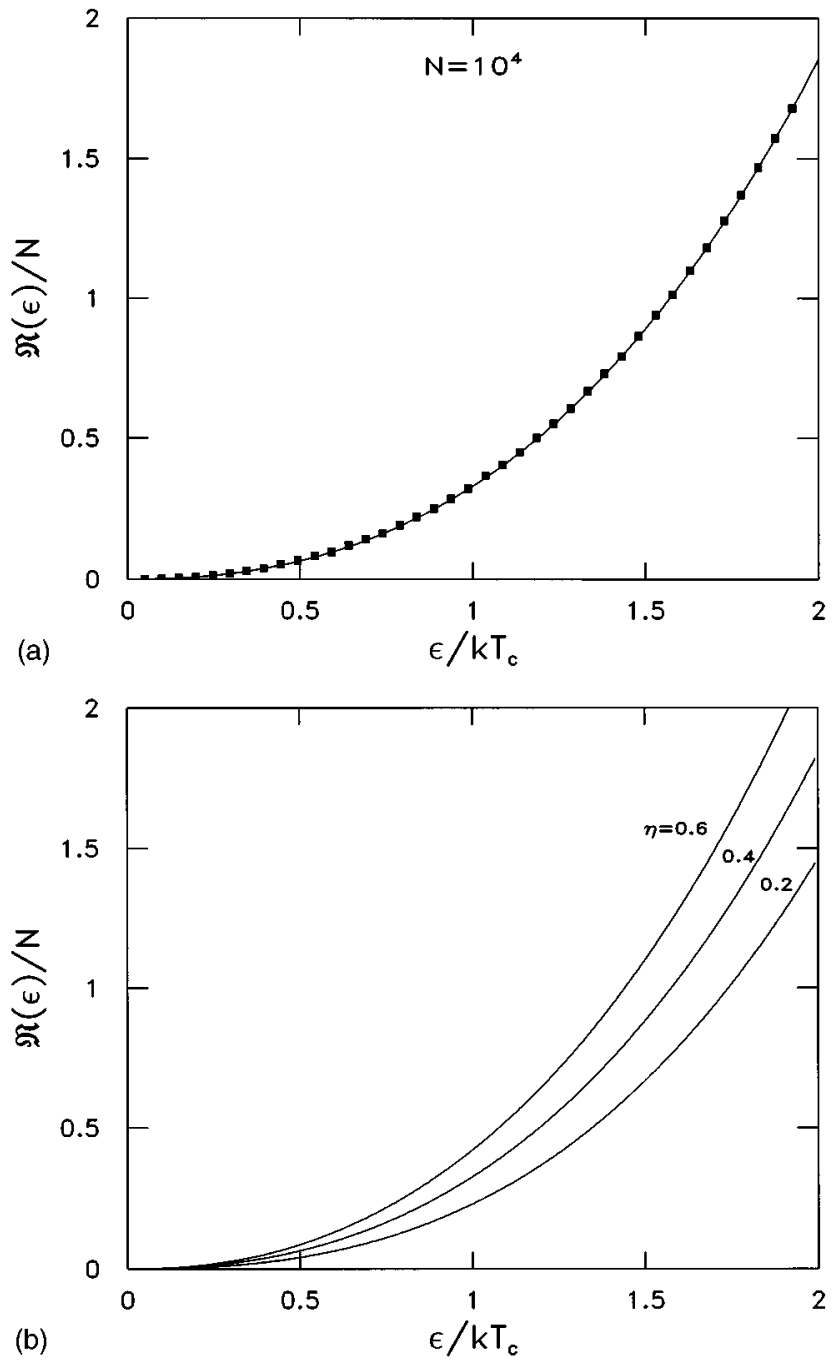

FIG. 5. Ratio $\mathfrak{N}(\epsilon) / N$ vs $\epsilon$, in units $k_{B} T_{c}$. In (a), the squares correspond to counting the states in the Bogoliubov spectrum of Fig. 1, while the solid line is the corresponding semiclassical approximation (13). The latter is indistinguishable from the formula (16), valid in the scaling regime $N a / a_{\mathrm{HO}} \gg 1$. In (b), the semiclassical prediction (16) is given for different values of the scaling parameter $\eta$.

corresponds to the semiclassical dispersion law. Here the quantity $n_{0}(\mathbf{r})=\Psi_{0}^{2}(\mathbf{r})$ is the condensate density. In Fig. 5(a) we compare the semiclassical result for $\mathfrak{N}(\epsilon) / N$ (solid line) with the one obtained from Eqs. (4) and (5) (squares) for $10^{4}$ atoms of rubidium in the same trap of Fig. 2. Here the energy is given in units $k_{B} T_{c}=\hbar \omega_{\mathrm{HO}}[N / \zeta(3)]^{1 / 3}$, which is the critical temperature for an ideal Bose gas in a harmonic trap; the value for $10^{4}$ atoms is $k_{B} T_{c}=20.26 \hbar \omega_{\mathrm{HO}}$ as shown also in Fig. 1. The accuracy of the semiclassical approximation turns out to be very high also for relatively low values of $\epsilon$.

The use of the semiclassical approximation allows one to carry out the analysis of the density of states in a systematic way and to exploit the dependence on the relevant parameters of the system. In fact, when the number of atoms in the condensate is large enough to make the Thomas-Fermi approximation (6) accurate, the statistical properties of the system can be expressed in terms of a single scaling parameter $\eta$ given by the ratio [22] 


$$
\eta=\frac{\mu^{\mathrm{TF}}}{k_{B} T_{c}}=1.57\left(\frac{N^{1 / 6} a}{a_{\mathrm{HO}}}\right)^{2 / 5}
$$

between the chemical potential (7), calculated at zero temperature in the Thomas-Fermi approximation, and the critical temperature $k_{B} T_{c}$. The ratio $\eta$ depends on the deformation of the trap only through the geometrical average of the os- cillator frequencies $\omega_{\mathrm{HO}}=\left(\omega_{x} \omega_{y} \omega_{z}\right)^{1 / 3}$. The parameters used in the calculation of Fig. 5(a) correspond to a spherical trap with $\eta=0.407$. As pointed out in Ref. [22], quite different experimental conditions (shape of the trap, value of $N$, etc.) can correspond to very similar values of $\eta$. In terms of the scaling parameter $\eta$ and the dimensionless energy $\widetilde{\epsilon}=\epsilon /\left(k_{B} T_{c}\right)$, the number of states $\mathfrak{N}(\widetilde{\epsilon})$ predicted by the Bogoliubov semiclassical theory becomes

$$
\frac{\mathfrak{N}(\widetilde{\boldsymbol{\epsilon}})}{N}=\int_{0}^{\widetilde{\epsilon}} d \widetilde{\boldsymbol{\epsilon}}^{\prime} \frac{4}{\pi \zeta(3)} \int_{0}^{1} d x \sqrt{1-x}\left[\widetilde{\boldsymbol{\epsilon}}^{\prime} \eta \frac{\sqrt{\left[x^{2}+\left(\tilde{\boldsymbol{\epsilon}}^{\prime}\right)^{2} / \eta^{2}\right]^{1 / 2}-x}}{\sqrt{x^{2}+\left(\tilde{\boldsymbol{\epsilon}}^{\prime}\right)^{2} / \eta^{2}}}+\left(\widetilde{\boldsymbol{\epsilon}}^{\prime}\right)^{2} \sqrt{x+\eta / \widetilde{\epsilon}^{\prime}}\right] .
$$

This result has been obtained by using the Thomas-Fermi approximation (6) for the condensate density in Eqs. (13) and (14); this allows one to split the space integral into an inside region (first term in the square bracket) and an outside region (second term). It is worth stressing that Eq. (16), which is expected to hold in the scaling regime $N a / a_{\mathrm{HO}} \gg 1$, provides a very good estimate of the semiclassical expression (13) even for relatively small $N$. For instance, the two predictions are indistinguishable in Fig. 5(a), being represented by the same solid line. In Fig. 5(b) we show the predictions for the density of states given by the semiclassical approximation (16) for three different values of $\eta$. The parameters of the recent experiments at Jila [1] and MIT [2], using very different traps, correspond to $\eta$ ranging from 0.39 to 0.45 .

Expression (16) can be also expanded at low energy, $\epsilon \ll k_{B} T_{c}$, still compatible with the assumption $\epsilon \gg \hbar \omega_{\mathrm{HO}}$. One finds the law $\mathfrak{N}(\epsilon) / N \propto \epsilon^{5 / 2}$. This differs from the usual $\epsilon^{3}$ law typical of the phonon regime, revealing the different behavior exhibited by these systems with respect to the homogeneous Bose gas.

\section{QUANTUM DEPLETION}

In the last part of the paper we calculate the quantum depletion of the condensate, which, according to Bogoliubov theory, is given by

$$
\frac{\delta N}{N}=\frac{1}{N} \sum_{j} \int d \mathbf{r}\left|v_{j}(\mathbf{r})\right|^{2}
$$

The "hole" components $v_{j}$ can be obtained by solving Eqs. (4) and (5). In the semiclassical approximation [9] one replaces the sum over all the discrete states with the integral over $\mathbf{p}$ of the function

$$
v^{2}(\mathbf{p}, \mathbf{r})=\frac{1}{2 \epsilon(\mathbf{p}, \mathbf{r})}\left(\frac{p^{2}}{2 m}+V_{\mathrm{ext}}(\mathbf{r})-\mu+2 g n_{0}(\mathbf{r})+\boldsymbol{\epsilon}(\mathbf{p}, \mathbf{r})\right)
$$

where $\epsilon(\mathbf{p}, \mathbf{r})$ is the single-particle energy (14). In a uniform gas this expression yields the most famous result $\delta N / N=(8 / 3)\left(n_{0} a^{3} / \pi\right)^{1 / 2}$. In the trapped gas and in the limit
$N a / a_{\mathrm{HO}} \gg 1$, where the TF approximation holds, the semiclassical approximation provides the simple analytic law [9]

$$
\frac{\delta N}{N}=\frac{\eta^{3}}{6 \sqrt{2} \zeta(3)}=0.098 \eta^{3}
$$

with $\eta$ given in Eq. (15). Since the available experiment corresponds to $\eta \simeq 0.4$, the quantum depletion turns out to be less than 1\%, as already pointed out in Refs. [5,7]. In Fig. 6 we show the quantum depletion for 10000 and 50000 atoms of rubidium obtained by summing over the Bogoliubov spectrum up to a given energy $\epsilon$ (solid lines). We compare it with the prediction of the semiclassical expression (18) (dashed lines), while the arrows indicate the asymptotic values (19), holding in the scaling limit. An important result emerging from the numerical calculation is the very slow convergence of the sum (17). This is not a surprise, since also in a homogeneous gas the convergence is slow due to the $1 / p^{4}$ tail in

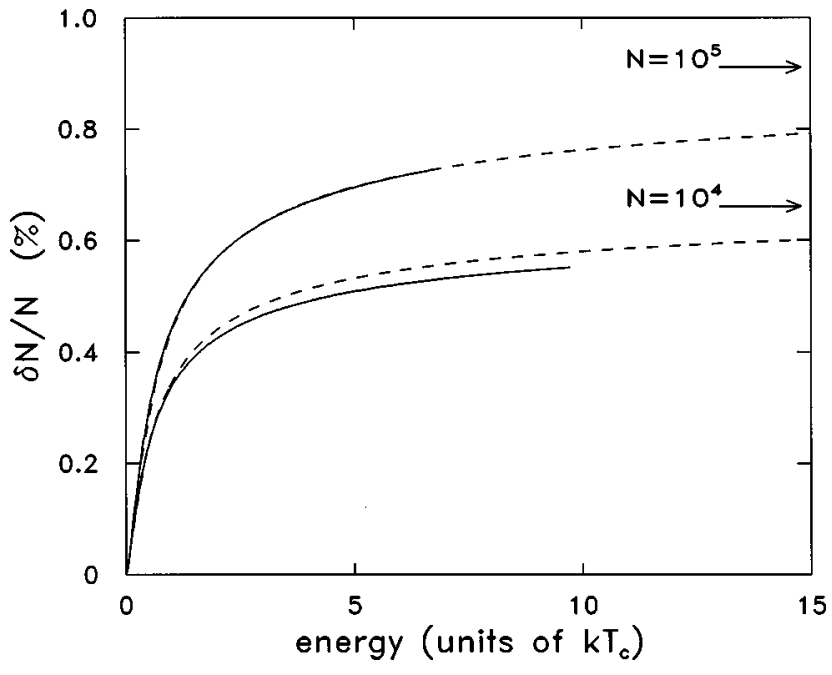

FIG. 6. Quantum depletion for 10000 (two lower curves) and 50000 (two upper curves) atoms of ${ }^{87} \mathrm{Rb}$ in a spherical trap with $a_{\mathrm{HO}}=0.791 \times 10^{-4} \mathrm{~cm}$. The depletion is plotted as a function of the maximum energy considered in the sum (17). Solid lines: $|v|^{2}$ from the solution of Eqs. (4) and (5); dashed lines: from the semiclassical approximation (18). Arrows: asymptotic scaling values (19). 
the momentum distribution at high momenta and one has to go up to $\epsilon=100 \mu$ in order to saturate $90 \%$ of the sum (17).

The agreement between the quantum depletion obtained from the discretized sum (17) over the Bogoliubov states and from the semiclassical approximation (18) is satisfying and was not obvious a priori. Figure 6 shows a discrepancy of the order of $5 \%$ between the two predictions for $N=10000$, while for larger $N$ the two curves tend to coincide. It is worth noticing that the two solid lines in Fig. 6 require the summation of $\int d \mathbf{r}\left|v_{j}(\mathbf{r})\right|^{2}$ over up to 15000 different values of $(n, \ell)$ in the Bogoliubov spectrum; the calculation is then much heavier than the semiclassical one. The good accuracy of the semiclassical approach makes it useful in practical situations. This is especially true for the simple formula (19), which includes the case of anisotropic traps through the averaged frequency $\omega_{\mathrm{HO}}=\left(\omega_{x} \omega_{y} \omega_{z}\right)^{1 / 3}$ entering the ThomasFermi chemical potential $\mu^{\mathrm{TF}}$ and, hence, the scaling parameter $\eta$.

Finally, the large $N$ semiclassical formula (19) shows the rather strong dependence of the depletion on the scattering length parameter $a$. If the magnetic tuning of the scattering length will become available, it will be possible in the future to increase significantly the value of $\eta$ and consequently explore Bose gases where the quantum depletion is much larger.

\section{SUMMARY}

We have investigated the elementary excitations of a dilute Bose gas in harmonic trap by solving the equations of Bogoliubov theory. Different from the case of a uniform gas, where phonons dominate the system at energies of the order of or lower than the chemical potential, the spectrum of the trapped gas shows an important particlelike behavior even at low energy. This fact has been here explored in detail. We have compared the results of Bogoliubov theory for the density of states with the ones of Hartree-Fock theory, finding a very good agreement on a wide range of energy. We have studied the behavior of surface modes, emphasizing the crossover from the low-energy regime, well described by the hydrodynamic model, to the single-particle regime. This crossover provides also a critical frequency associated with a rotational instability and we have compared this frequency with the one needed to create a quantized vortex. Another important result emerging from our analysis is the high accuracy exhibited by the semiclassical approximation for the excited states. Finally, we have calculated the quantum depletion of the condensate by summing the "hole" component $\int d \mathbf{r}|v(\mathbf{r})|^{2}$ over all the states in the excitation spectrum of Bogoliubov theory. The convergence of the sum turns out to be very slow, as expected by the analogy with the case of the uniform gas. Again we find excellent agreement with the predictions of the semiclassical approximation. In the limit $N a / a_{\mathrm{HO}} \gg 1$, the latter provides the simple and useful formula $\delta N / N=0.098 \eta^{3}$, in terms of the scaling parameter $\eta=1.57\left(N^{1 / 6} a / a_{\mathrm{HO}}\right)^{2 / 5}$.

\section{ACKNOWLEDGMENTS}

We thank C. J. Pethick for useful discussions about the behavior of the surface excitations. M.G. thanks the Ministerio de Educación y Ciencia (Spain) for financial support. L.P. would like to acknowledge the hospitality of the Dipartimento di Fisica, Università di Trento and the financial support of the Istituto Nazionale per la Fisica della Materia.
[1] D. S. Jin, J. R. Ensher, M. R. Matthews, C. E. Wieman, and E. A. Cornell, Phys. Rev. Lett. 77, 420 (1996).

[2] M.-O. Mewes, M. R. Andrews, N. J. van Druten, D. M. Kurn, D. S. Durfee, C. G. Townsend, and W. Ketterle, Phys. Rev. Lett. 77, 988 (1996).

[3] S. Stringari, Phys. Rev. Lett. 77, 2360 (1996).

[4] M. Edwards, P. A. Ruprecht, K. Burnett, R. J. Dodd, and C. W. Clark, Phys. Rev. Lett. 77, 1671 (1996); P. A. Ruprecht, Mark Edwards, K. Burnett, and Charles W. Clark, Phys. Rev. A 54, 4178 (1996); M. Edwards, R. J. Dodd, C. W. Clark, and K. Burnett, J. Res. Natl. Inst. Stand. Technol. 101, 553 (1996).

[5] K. G. Singh and D. S. Rokhsar, Phys. Rev. Lett. 77, 1667 (1996).

[6] B. D. Esry, Phys. Rev. A 55, 1147 (1997).

[7] D. A. W. Hutchinson, E. Zaremba, and A. Griffin, Phys. Rev. Lett. 78, 1842 (1997).

[8] L. You, W. Hoston, and M. Lewenstein, Phys. Rev. A 55, R1581 (1997).

[9] S. Giorgini, L. P. Pitaevskii, and S. Stringari, J. Low Temp. Phys. (to be published).

[10] L. P. Pitaevskii, Zh. Eksp. Teor. Fiz.40, 646 (1961) [Sov. Phys. JETP 13, 451 (1961)]; E. P. Gross, Nuovo Cimento 20, 454 (1961); E. P. Gross, J. Math. Phys. (N.Y.) 4, 195 (1963).
[11] A. L. Fetter, Phys. Rev. A 53, 4245 (1996); A. L. Fetter and D. Rokhsar, e-print cond-mat/9704234.

[12] W.-C. Wu and A. Griffin, Phys. Rev. A 54, 4204 (1996).

[13] V. V. Goldman, I. F. Silvera, and A. J. Leggett, Phys. Rev. B 24, 2870 (1981); D. A. Huse and E. D. Siggia, J. Low Temp. Phys. 46, 137 (1982).

[14] Actually Hamiltonian (11) ignores nonlocality effects that modify mainly the $\ell=0$ solutions of the Hartree-Fock theory. These states are, however, irrelevant for the determination of the density of states.

[15] C. Pethick (private communication).

[16] G. Baym and C. Pethick, Phys. Rev. Lett. 76, 6 (1996).

[17] F. Dalfovo, L. Pitaevskii, and S. Stringari, Phys. Rev. A 54, 4213 (1996).

[18] E. Lundh, C. J. Pethick, and H. Smith, Phys. Rev. A 55, 2126 (1997).

[19] F. Dalfovo and S. Stringari, Phys. Rev. A 53, 2477 (1996).

[20] S. Giorgini, L. Pitaevskii, and S. Stringari, Phys. Rev. A 54, 4633 (1996).

[21] E. Timmermans, P. Tommasini, and K. Huang, Phys. Rev. A 55, 3645 (1997).

[22] S. Giorgini, L. Pitaevskii, and S. Stringari, Phys. Rev. Lett. 78, 3987 (1997). 